\title{
ERG gene rearrangements are common in prostatic small cell carcinomas
}

Tamara L Lotan ${ }^{1}$, Nilesh S Gupta ${ }^{2}$, Wenle Wang ${ }^{1}$, Antoun Toubaji ${ }^{1}$, Michael C Haffner ${ }^{3}$, Alcides Chaux ${ }^{1}$, Jessica L Hicks ${ }^{1}$, Alan K Meeker ${ }^{1}$, Charles J Bieberich ${ }^{4}$, Angelo M De Marzo ${ }^{1,3,5}$, Jonathan I Epstein ${ }^{1,3,5}$ and George J Netto ${ }^{1,3,5}$

${ }^{1}$ Department of Pathology, Johns Hopkins Medical Institutions, Baltimore, MD, USA; ${ }^{2}$ Department of Pathology, Henry Ford Hospital, Detroit, MI, USA; ${ }^{3}$ Department of Oncology, Johns Hopkins Medical Institutions, Baltimore, MD, USA; ${ }^{4}$ Department of Biological Sciences, University of Maryland Baltimore County, Baltimore, MD, USA and ${ }^{5}$ Department of Urology, Johns Hopkins Medical Institutions, Baltimore, $M D, U S A$

Small cell carcinoma of the prostate is a rare subtype with an aggressive clinical course. Despite the frequent occurrence of ERG gene rearrangements in acinar carcinoma, the incidence of these rearrangements in prostatic small cell carcinoma is unclear. In addition, molecular markers to distinguish prostatic small cell carcinomas from lung and bladder small cell carcinomas may be clinically useful. We examined the occurrence of ERG gene rearrangements by fluorescence in situ hybridization in prostatic, bladder and lung small cell carcinomas. We also examined the expression of ERG, androgen receptor (AR) and NKX3-1 by immunohistochemistry in prostatic cases. Overall, $45 \%(10 / 22)$ of prostatic small cell carcinoma cases harbored ERG rearrangements, whereas no cases of bladder or lung small cell carcinomas showed ERG rearrangement (0/12 and 0/13, respectively). Of prostatic small cell carcinoma cases, $80 \%(8 / 10)$ showed ERG deletion and $20 \%(2 / 10)$ showed ERG translocation. In $83 \%(5 / 6)$ of prostatic small cell carcinoma cases in which a concurrent conventional prostatic acinar carcinoma component was available for analysis, there was concordance for the presence/absence of ERG gene rearrangement between the different subtypes. ERG, AR and NKX3-1 protein expression was detected in a minority of prostatic small cell carcinoma cases $(23,27$ and $18 \%$, respectively), while these markers were positive in the majority of concurrent acinar carcinoma cases $(66,83$ and $83 \%$, respectively). The presence of ERG rearrangements in nearly half of the prostatic small cell carcinomas is a similar rate of rearrangement to that found in prostatic acinar carcinomas. Furthermore, the high concordance rate of $E R G$ rearrangement between the small cell and acinar components in a given patient supports a common origin for these two subtypes of prostate cancer. Finally, the absence of $E R G$ rearrangement in bladder or lung small cell carcinomas highlights the utility of detecting ERG rearrangement in small cell carcinomas of unknown primary for establishing prostatic origin.

Modern Pathology (2011) 24, 820-828; doi:10.1038/modpathol.2011.7; published online 18 February 2011

Keywords: androgen receptor; ERG; gene fusion; NKX3-1; prostatic adenocarcinoma; small cell carcinoma; TMPRSS2-ERG

Small cell carcinoma of the prostate is an unusual and aggressive subtype, accounting for $0.5-2 \%$ of all prostate cancers. ${ }^{1,2}$ Typically, the diagnosis is made using morphological criteria as most small cell

Correspondence: Dr GJ Netto, MD, Johns Hopkins Medical Institutions, 401 North Broadway, Weinberg Building, Suite 2242, Baltimore, MD 21231, USA.

E-mail: gnetto1@jhmi.edu

Received 28 June 2010; revised 3 January 2011; accepted 3 January 2011; published online 18 February 2011 carcinomas of prostatic origin share the classic 'oat-cell' morphology with those described in the lung. Approximately $50 \%$ of the time, prostatic small cell carcinoma occurs concurrently with usual-type acinar carcinoma and the two components may be intermingled, thus suggesting a common origin; however, molecular evidence for clonal evolution has been limited. ${ }^{1-3}$ When prostatic small cell carcinomas occur without an associated acinar component, particularly in a metastatic site, it can be difficult to determine the site of origin of 
the tumor. Several recent studies have shown that immunohistochemical markers of prostatic origin, including PSA, PSAP, PSMA and p501s, can be negative in up to $80 \%$ of small cell carcinoma cases. $^{4-6}$ Further complicating the situation, TTF-1, a common marker of lung carcinoma is positive in $>50 \%$ of cases. ${ }^{5,6}$

Recently, a bioinformatics approach uncovered a gene rearrangement present in $40-60 \%$ of conventional prostatic acinar carcinoma cases, making it the most common rearrangement identified in human cancer to date. ${ }^{7}$ This rearrangement occurs between an androgen-regulated gene, TMPRSS2 (21q22.3) and an ETS transcription factor family member, most commonly ERG (21q22.2), resulting in a gene fusion product. ${ }^{7-9}$ This gene fusion can occur through a small deletion on chromosome 21 (seen in approximately two-thirds of acinar cases displaying ERG rearrangement) or through a translocation. ${ }^{9,10}$ Although the clinicopathological significance of this genetic rearrangement has remained controversial, it is clear that ERG rearrangements are specific and sensitive for prostatic acinar carcinomas. ${ }^{11-15}$

Despite the numerous studies of TMPRSS2-ERG gene fusions in prostatic adenocarcinomas, only one recent small study has documented the occurrence of this rearrangement in androgen-independent metastases of prostatic small cell carcinomas. ${ }^{16}$ However, data on the rates of ERG rearrangement in primary prostatic small cell carcinomas and the concordance of ERG rearrangements in concurrent small cell and acinar carcinoma tumors are lacking. In this study, we studied the rate of ERG gene rearrangement in 29 cases of primary prostatic small cell carcinomas by fluorescence in situ hybridization (FISH). Given recent reports that establish an etiological relationship between the androgensignaling axis and ERG rearrangement, we also examined the expression of ERG protein, androgen receptor (AR) and NKX3-1 (an androgen-regulated transcription factor), in these cases. ${ }^{17-19}$

\section{Materials and methods}

\section{Tissue Selection}

A tissue microarray was manually constructed from 29 specimens with prostatic small cell carcinomas retrieved from the surgical pathology and consultation files of the Johns Hopkins Hospitals from 1994 to 2008. In each case, a minimum of three $1.0-\mathrm{mm}$ cores were punched from the small cell carcinoma component, the acinar carcinoma component (when present) and the paired benign prostatic tissue, with 3-18 cores from each patient represented on the array. A separate tissue microarray was constructed from 12 bladder small cell carcinoma cases and from 13 lung small cell carcinoma cases, with each case arrayed in triplicate in $1.5-\mathrm{mm}$ cores.

\section{Fluorescence In Situ Hybridization}

FISH was performed on the tissue microarray, using break-apart probe for $5^{\prime} E R G$ and $3^{\prime} E R G$. In brief, a paraffin section of $4 \mu \mathrm{m}$-thickness was baked at $56^{\circ} \mathrm{C}$ for $2 \mathrm{~h}$, and then dewaxed and rehydrated using xylene and graded ethanol, respectively. The tissue microarray section was pretreated using the paraffin pretreatment reagent kit III (Abbott Molecular; Abbott Park, IL). BAC FISH probes were SpectrumGreen d-UTP direct-labeled BAC RP11-95I21 for $5^{\prime} E R G$ and SpectrumOrange d-UTP direct-labeled BAC RP11-476D17 for 3'ERG (Nick transKit, Vysis, Abbott Park). Tissue microarrays and BAC FISH probes were co-denatured at $94^{\circ} \mathrm{C}$ for $5 \mathrm{~min}$ and hybridized overnight at $37^{\circ} \mathrm{C}$ in a humid chamber (StatSpin ThermoBrite; IRIS, MA).

\section{FISH Scoring}

FISH scoring was conducted using a Nikon 50i epifluorescence microscope equipped with an X-Cite series 120 illuminator (EXFO Photonics Solutions, Ontario, CA) and a $\times 100 / 1.4$ NA oil immersion Neofluar lens. Fluorescence excitation/ emission filters were as follows: Cy3 excitation, 546 nm/10 nm BP; emission, 578 nm LP (Carl Zeiss); DAPI excitation, $330 \mathrm{~nm}$; emission, $400 \mathrm{~nm}$ using an XF02 fluorescence set (Omega Optical, Brattleboro, VT); Alexa Fluor 488 excitation, $475 \mathrm{~nm}$; emission, $535 \mathrm{~nm}$ using a combination of 475RDF40 and 535RDF45 filters (Omega Optical). Gray-scale images were captured for presentation using Nikon NIS-Elements software and an attached Photometrics CoolsnapEZ digital camera, pseudo-colored and merged.

\section{FISH Interpretation}

FISH interpretation was performed by a urological pathologist (TLL).

In each case, a minimum of 50 cells were scored for the presence/absence of $E R G$ gene fusion through deletion or translocation as follows: A nucleus without ERG rearrangement shows two pairs of juxtaposed red and green signals (Figure 1, top panels). A nucleus with ERG rearrangement through translocation shows one or more pairs of the red and green signals split apart and spatially separated in different regions of the nucleus (Figure 1, bottom panels). Finally, a nucleus with ERG rearrangement through deletion shows an absence of one or more green signals (Figure 2). Any case with one of the above ERG signal abnormalities in $\geq 10 \%$ of the nuclei in $\geq 1$ tissue microarray spot was scored as a fusion case and classified accordingly. Digitally scanned adjacent hematoxylin and eosin serial sections were available for side-by-side comparison with the FISH image to localize tumor cells and to confirm small cell carcinoma morphology. Four 

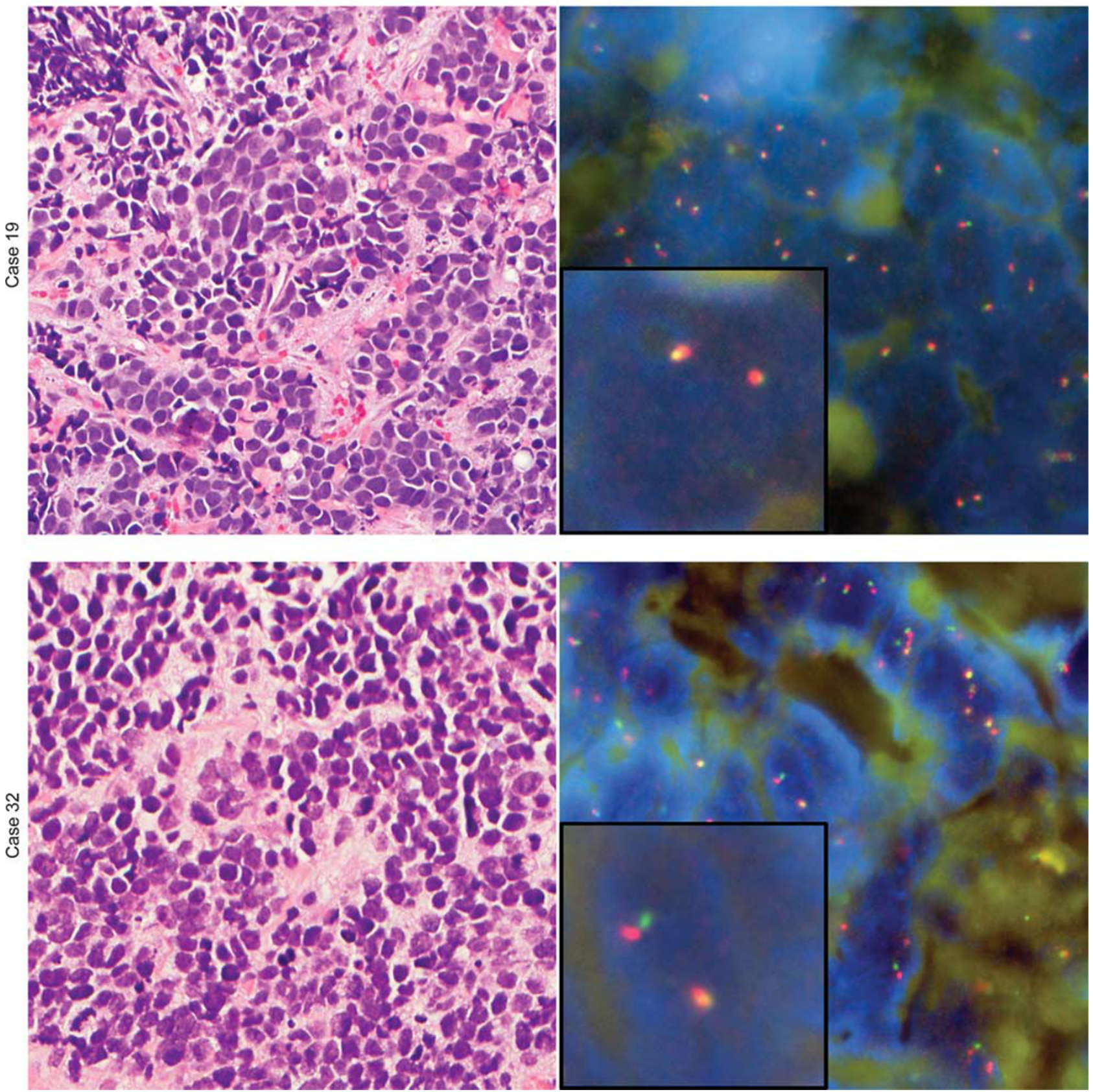

Figure 1 Top left: High-power photomicrograph ( $\times 400$ magnification) of a hematoxylin and eosin-stained section of small cell carcinoma case 19. Top right: Fluorescence in situ hybridization (FISH) $(\times 1000$ magnification) reveals no evidence of ERG rearrangement in this case. Bottom left: High-power photomicrograph $(\times 400$ magnification $)$ of a hematoxylin and eosin-stained section of small cell carcinoma case 32. Bottom right: FISH ( $\times 1000$ magnification) reveals ERG rearrangement through translocation with one juxtaposed red-green (yellow) signal in each nucleus and the second pair of red and green signals split apart and spatially separated in different regions of the nucleus.

cases of the benign prostatic epithelium were scored on the tissue microarray as a negative control.

\section{Immunohistochemistry}

Tissue microarray sections were deparaffinized, rehydrated and briefly equilibrated in water. Antigen unmasking was performed by steaming in citrate buffer (pH 6.0) for $25 \mathrm{~min}$ (NKX3-1, ERG) or HTTR (Target Retrieval Solution; Dako, Glostrup, Denmark) for $50 \mathrm{~min}$ (AR). Endogenous peroxidase activity was quenched by incubation with a peroxidase block for $5 \mathrm{~min}$ at room temperature. Nonspecific binding was blocked by incubating in $1 \%$ bovine serum albumin in Tris- $\mathrm{HCl} \mathrm{pH} 7.5$ for 
$H \& E$
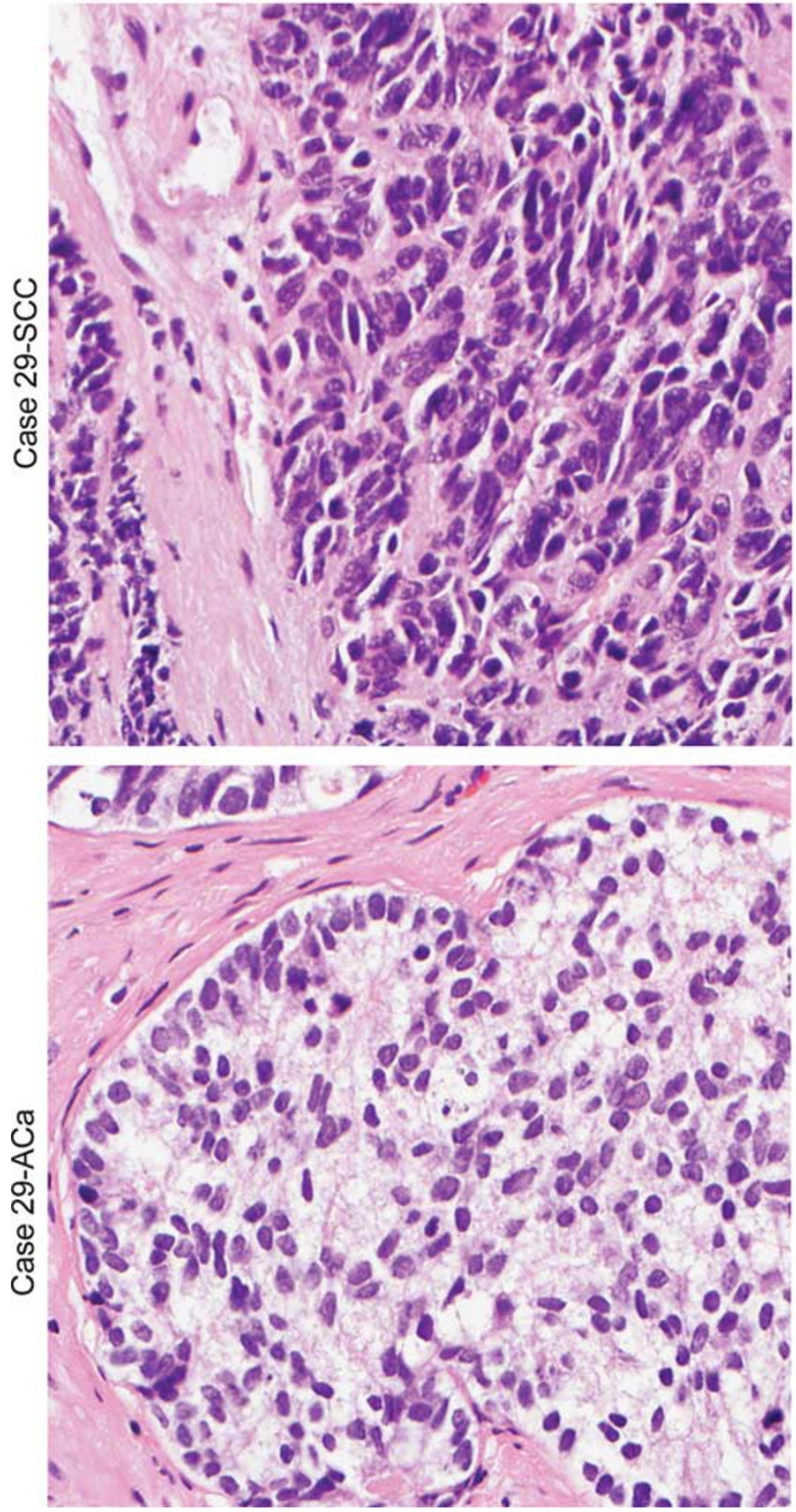

ERG FISH
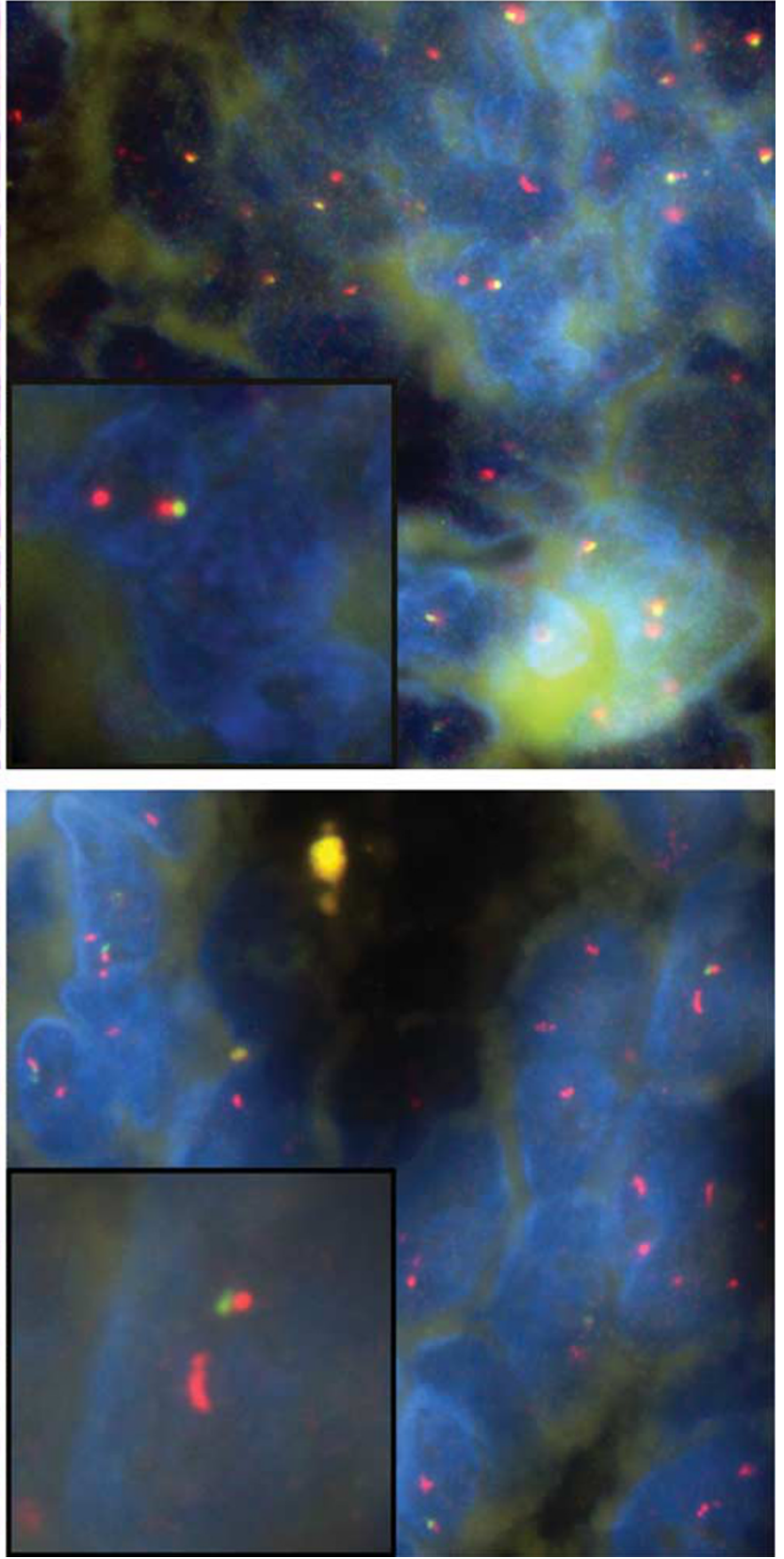

Figure 2 Top left: High-power photomicrograph ( $\times 400$ magnification) of a hematoxylin and eosin-stained section of small cell carcinoma component of case 29. Top right: Fluorescence in situ hybridization (FISH, $\times 1000$ magnification) reveals ERG rearrangement through deletion in the small cell component, with one juxtaposed red-green (yellow) signal in each nucleus and the absence of the second green signal. Bottom left: High-power photomicrograph ( $\times 400$ magnification) of a hematoxylin and eosin-stained section of the acinar carcinoma component of case 29. Bottom right: FISH ( $\times 1000$ magnification) reveals $E R G$ rearrangement through deletion in the acinar component as well.

20 min at room temperature. Slides were incubated with rabbit polyclonal anti-human NKX3-1 antibody (1:1000 dilution ${ }^{20}$ ), a rabbit monoclonal anti-human ERG antibody (Epitomics, Burlingame, CA; 1:75 dilution) or a rabbit polyclonal anti-human AR antibody (Santa Cruz Biotechnology, Santa Cruz, CA; 1:1000 dilution) for $45 \mathrm{~min}$ at room temperature. A horseradish peroxidase-labeled polymer (PowerVision, Leica Microsystems, Bannockburn, IL) was applied for $30 \mathrm{~min}$ at room temperature. Signal detection was performed using 3,3'-diaminobenzidine tetrahydrochloride as the chromagen. Slides were counterstained with hematoxylin, dehydrated and mounted.

\section{Interpretation of Immunohistochemistry}

ERG, AR and NKX3-1 immunostains were scored in small cell carcinoma and the associated acinar 
carcinoma component by a urological pathologist (TLL). Each spot was scored as positive if $>10 \%$ of cells showed nuclear staining ( 1 to $3+$ ) with either marker. A case was considered positive for each marker if any of the spots from that case were positive.

\section{Results}

In all, 22 of the 29 small cell carcinoma cases were evaluable for $E R G$ gene rearrangements $(76 \%)$. Of these, $15(68 \%)$ were transurethral resections of the prostate, $4(18 \%)$ were bladder biopsies, 2 (9\%) were prostate needle biopsies and $1(4 \%)$ was a radical prostatectomy. The average patient age was 73 years

Table 1 Frequency of ERG rearrangement by deletion and translocation in small cell carcinoma cases and concurrent acinar carcinoma

\begin{tabular}{lcc}
\hline & $\begin{array}{c}\text { Small cell } \\
\text { carcinoma cases }\end{array}$ & $\begin{array}{c}\text { Concurrent acinar } \\
\text { carcinoma component }\end{array}$ \\
\hline Total cases & 22 & 6 \\
Rearrangement & $10(45 \%)$ & $4(66 \%)$ \\
Deletion & $8(80 \%)$ & $4(100 \%)$ \\
Translocation & $2(20 \%)$ & $0(0 \%)$ \\
\hline
\end{tabular}

(range: 57-93 years). The diagnosis of small cell carcinoma of prostatic origin was confirmed by a concurrent or previous history of prostatic acinar carcinomas in $86 \%(19 / 22)$ of cases, whereas in the remainder, it was established by positive PSA immunostaining in the small cell tumor $(5 \%, 1 / 22)$ or by a documented negative cystoscopy $(9 \%, 2 / 22)$ to rule out a small cell carcinoma of urothelial origin. Overall, $45 \%(10 / 22)$ of prostatic small cell carcinoma cases had ERG gene rearrangements, with $80 \%(8 / 10)$ showing deletion and $20 \%(2 / 10)$ showing translocation (Table 1, Figures 1 and 2). In $27 \%(6 / 22)$ of small cell carcinoma cases, a concurrent acinar carcinoma component was available on the tissue microarray for analysis. Overall, $66 \%(4 / 6)$ cases had a Gleason score of $\geq 9$ in the acinar component, whereas $33 \%(2 / 6)$ cases had a Gleason score of $7-8$. In $83 \%$ (5/6) of the concurrent acinar carcinoma components, there was concordance for the presence/absence of $E R G$ gene rearrangement between the different histological subtypes (Figure 2 and Table 2). Overall, 50\% (3/6) of these cases showed concurrent ERG deletion in both subtypes, and $17 \%$ (1/6) of cases showed an $E R G$ translocation in the small cell carcinoma component and an $E R G$ deletion in the adenocarcinoma component. In all, 33\% (2/6) did not show evidence of $E R G$ rearrangement in either component.

Table 2 Frequency of ERG rearrangement by fluorescence in situ hybridization (FISH) and ERG, NKX3-1 and AR protein expression by immunohistochemistry in small cell carcinoma (S) cases and the concurrent acinar carcinoma (A) component

\begin{tabular}{|c|c|c|c|c|c|c|c|}
\hline $\begin{array}{l}\text { Case } \\
\text { no. }\end{array}$ & Specimen type & $\begin{array}{l}\text { Prostatic small cell } \\
\text { carcinoma confirmed by }\end{array}$ & $\begin{array}{l}\text { Tumor } \\
\text { type }\end{array}$ & FISH & $\begin{array}{l}E R G \\
\text { protein }\end{array}$ & $\begin{array}{c}A R \\
\text { protein }\end{array}$ & $\begin{array}{l}\text { NKX3-1 } \\
\text { protein }\end{array}$ \\
\hline \multirow[t]{2}{*}{1} & TURP & Concurrent acinar carcinoma & $\mathrm{S}$ & $\mathrm{N}$ & 0 & 0 & 0 \\
\hline & & & A & $\mathrm{N}$ & 0 & + & + \\
\hline 5 & TURP & Concurrent acinar carcinoma & $\mathrm{S}$ & $\mathrm{N}$ & 0 & 0 & 0 \\
\hline \multirow[t]{2}{*}{8} & TURP & Concurrent acinar carcinoma & $\mathrm{S}$ & $\mathrm{D}$ & + & 0 & 0 \\
\hline & & & A & $\mathrm{D}$ & + & + & + \\
\hline 10 & TURP & Concurrent acinar carcinoma & $\mathrm{S}$ & $\mathrm{N}$ & 0 & 0 & 0 \\
\hline 12 & Prostate biopsy & Negative cystoscopy & S & $\mathrm{N}$ & 0 & 0 & 0 \\
\hline 14 & TURP & Concurrent acinar carcinoma & $\mathrm{S}$ & $\mathrm{N}$ & 0 & 0 & 0 \\
\hline 15 & TURP & PSA & S & $\mathrm{D}$ & + & + & + \\
\hline 16 & TURP & History of acinar carcinoma & $\mathrm{S}$ & $\mathrm{N}$ & 0 & 0 & 0 \\
\hline \multirow[t]{2}{*}{17} & Radical prostatectomy & Concurrent acinar carcinoma & $\mathrm{S}$ & $\mathrm{T}$ & 0 & + & 0 \\
\hline & & & A & $\mathrm{D}$ & + & + & + \\
\hline 18 & Prostate biopsy & Concurrent acinar carcinoma & $\mathrm{S}$ & $\mathrm{D}$ & 0 & 0 & 0 \\
\hline 19 & TURP & Negative cystoscopy & $\mathrm{S}$ & $\mathrm{N}$ & 0 & 0 & 0 \\
\hline 20 & Bladder biopsy & Concurrent acinar carcinoma & S & $\mathrm{N}$ & + & 0 & 0 \\
\hline 21 & TURP & Concurrent acinar carcinoma & $\mathrm{S}$ & $\mathrm{D}$ & 0 & 0 & 0 \\
\hline \multirow[t]{2}{*}{25} & Bladder biopsy & Concurrent acinar carcinoma & $\mathrm{S}$ & $\mathrm{N}$ & 0 & 0 & 0 \\
\hline & & & A & $\mathrm{N}$ & 0 & 0 & 0 \\
\hline 26 & TURP & Concurrent acinar carcinoma & $\mathrm{S}$ & $\mathrm{D}$ & + & + & + \\
\hline \multirow[t]{2}{*}{27} & TURP & Concurrent acinar carcinoma & $\mathrm{S}$ & $\mathrm{D}$ & + & + & + \\
\hline & & & A & $\mathrm{D}$ & + & + & + \\
\hline 28 & TURP & Concurrent acinar carcinoma & $\mathrm{S}$ & $\mathrm{N}$ & 0 & + & + \\
\hline \multirow[t]{2}{*}{29} & TURP & Concurrent acinar carcinoma & $\mathrm{S}$ & $\mathrm{D}$ & 0 & 0 & 0 \\
\hline & & & A & $\mathrm{D}$ & + & + & + \\
\hline 30 & Bladder biopsy & History of acinar carcinoma & $\mathrm{S}$ & $\mathrm{N}$ & 0 & + & 0 \\
\hline 31 & Bladder biopsy & History of acinar carcinoma & $\mathrm{S}$ & $\mathrm{N}$ & 0 & 0 & 0 \\
\hline 32 & TURP & History of acinar carcinoma & $\mathrm{S}$ & $\mathrm{T}$ & 0 & 0 & 0 \\
\hline 33 & TURP & History of acinar carcinoma & $\mathrm{S}$ & $\mathrm{D}$ & 0 & 0 & 0 \\
\hline
\end{tabular}

D, ERG rearrangement by deletion; N, no ERG rearrangement; +, positive; T, ERG rearrangement by translocation; TURP, transurethral resection of the prostate; 0 , negative. 


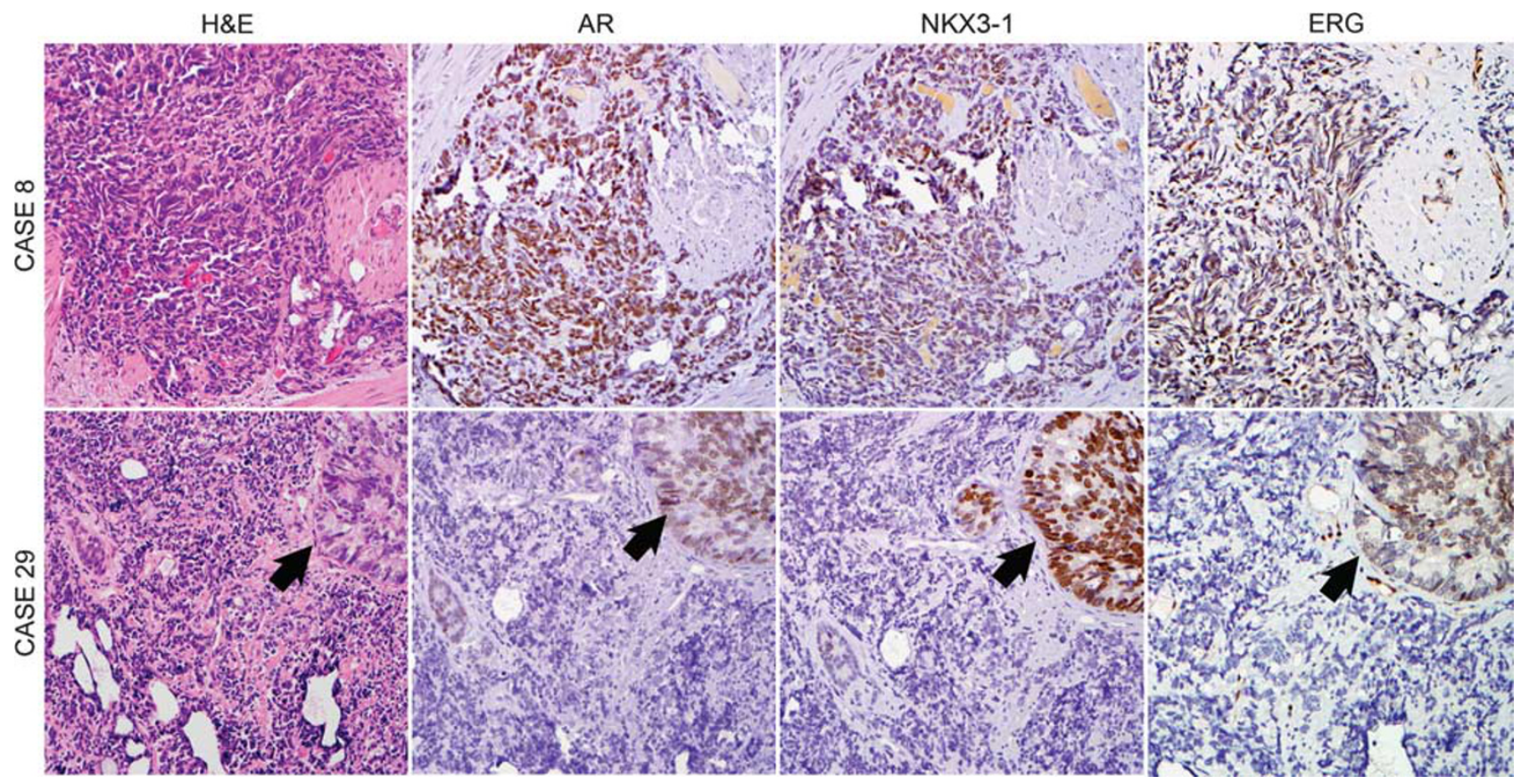

Figure 3 Top row: Small cell carcinoma case 8 is strongly and diffusely positive for the androgen receptor (AR), NKX3-1 and ERG protein. Bottom row: In contrast, case 29 shows negativity for all three markers in the small cell component, whereas the adjacent acinar component (arrows) is positive for all three markers.

ERG protein expression was detected in $23 \%$ (5/22) of prostatic small cell carcinoma cases (Table 2 and Figure 3). Of the ERG protein-positive cases, $80 \%$ (4/5) were concordant for $E R G$ gene rearrangement by FISH, with $100 \%(4 / 4)$ of these cases showing ERG deletion. The sensitivity of ERG protein expression for detecting prostatic small cell cases with ERG gene rearrangement was $40 \%$ (4/10 FISH-positive cases detected), whereas the specificity was 92\% (11/12 FISH-negative cases were ERG protein negative). Of cases with a concurrent acinar carcinoma component available for analysis, 66\% (4/6) were concordant in acinar and small cell components for ERG protein expression (2 cases positive and 2 cases negative), whereas 33\% (2/6) cases were discordant, with ERG protein expression in the acinar component and absent from the small cell component (Figure 3, bottom panels). In concurrent acinar tumors, ERG protein expression was $100 \%$ sensitive (4/4 FISH-positive cases detected) and $100 \%$ specific $(2 / 2$ FISH-negative cases were ERG protein negative) for $E R G$ rearrangement by FISH.

AR and NKX3-1 expression was detected in only a minority of prostatic small cell carcinoma cases ( $27 \%$ or $6 / 22$ and $18 \%$ or $4 / 22$, respectively, Figure 3 ). Although only a small fraction of cases expressed these markers, there was a high concordance between them, with 66\% (4/6) cases expressing AR also expressing NKX3-1 and 100\% (16/16) of cases negative for AR also negative for NKX3-1 $(P=0.0021$ by Fisher's exact test, Table 2). Interestingly, 75\% (3/4) of small cell carcinoma cases expressing $\mathrm{AR}$ and NKX3-1 showed ERG rearrangement (all by deletion), whereas only $44 \%$ (7/16) of the cases that were entirely negative for both AR and NKX3-1 showed $E R G$ rearrangement. ERG protein expression was only loosely correlated with AR status, as $60 \%(3 / 5)$ cases expressing the ERG protein were also AR positive and $82 \%(14 / 17)$ cases negative for the ERG protein were also negative for AR $(P=0.10005$ by Fisher's exact test, Table 2). In contrast to the rates of expression in small cell carcinomas, AR and NKX3-1 were diffusely and strongly positive in $83 \%(5 / 6)$ of concurrent acinar carcinoma cases, with only one case failing to express either marker. In several cases in which the acinar and small cell components were spatially juxtaposed, positivity for AR and NKX3-1 expression could be seen in the acinar component, whereas both were entirely absent from the small cell component (Figure 3, bottom panels).

Overall, 12 bladder small cell carcinoma cases were evaluable for $E R G$ gene rearrangements. Of these, $6(50 \%)$ were radical cystectomy or cystoprostatectomy specimens, $3(25 \%)$ were transurethral resections of bladder tumor (TURBT), 2 (17\%) were partial cystectomies and $1(8 \%)$ was a bladder biopsy. The average patient age was 66 years (range: 35-77), with 10 males and 2 females. The diagnosis of small cell carcinomas of bladder origin was confirmed by a concurrent or past history of conventional invasive urothelial carcinomas in each case. No cases of bladder small cell carcinomas (0/ 12) showed $E R G$ gene rearrangement by FISH.

In all, 13 lung small cell carcinoma cases were evaluable for $E R G$ gene rearrangements. Of these, 8 $(62 \%)$ were lobectomy specimens, $3(23 \%)$ were wedge resections and $2(15 \%)$ were bronchoscopic biopsies. The average patient age was 64 years 
(range: 49-80), with 7 males and 6 females. No cases of pulmonary small cell carcinomas $(0 / 13)$ showed $E R G$ gene rearrangement by FISH.

\section{Discussion}

The prostate is a relatively common site of origin for extrapulmonary small cell carcinomas. ${ }^{1,2,21}$ Neuroendocrine cells have long been recognized as a normal component of the benign prostate tissue, and depending on the method of detection, up to $100 \%$ of acinar adenocarcinomas can express some degree of neuroendocrine markers. ${ }^{22}$ Small cell carcinomas of the prostate differ significantly from usual prostatic acinar adenocarcinomas, both morphologically and immunophenotypically. Furthermore, distinguishing small cell carcinomas from highgrade (Gleason pattern 4 or 5) acinar prostate carcinomas is consequential for patient clinical management. Small cell carcinomas are extremely aggressive tumors, with patients typically presenting with urinary obstruction (in the current series, the majority of specimens were from transurethral resections) and often showing evidence of metastases at the time of diagnosis., ${ }^{2,23-29}$ In addition to their rapid growth, this may also be due to the fact that small cell carcinomas are less likely to be detected by PSA screening compared with their acinar carcinoma counterparts. ${ }^{2}$ Importantly, although small cell carcinomas are often resistant to androgen-deprivation therapy at the outset, they are at least temporarily sensitive to cisplatin-based chemotherapeutic regimens in contrast to acinar carcinomas. ${ }^{2,24-29}$

Despite these important clinical differences between prostatic small cell and acinar carcinomas, few studies have compared the two tumor types at the molecular genetic level, in part because of the lack of readily available molecular markers. The recent discovery of chromosomal rearrangements involving the $E R G$ locus in acinar prostatic adenocarcinoma presents the opportunity to compare the two prostate cancer phenotypes. Recently, four smaller studies have established that ERG rearrangements do exist in prostatic small cell carcinomas. ${ }^{16,30-32}$ Interestingly, one of the few prostate carcinoma cell lines found to harbor the rearrangement (also by deletion) is the NCI-H660 line, an ARnegative cell line derived from a small cell carcinoma metastasis. ${ }^{30}$ In another recent study, Han et $a 1^{16}$ examined 7 cases of metastatic small cell carcinomas from an androgen-independent metastatic autopsy cohort and found that $71 \%(5 / 7)$ of these cases harbored the ERG rearrangement, with all cases showing rearrangement by deletion. The authors suggest that the high prevalence of rearrangement by deletion in their series may correlate with earlier studies suggesting that rearrangement through deletion is associated with a more aggressive disease course compared with translocation.
Importantly, the rate of $E R G$ rearrangement in primary prostate tumors with small cell morphology has not been previously determined in a large series of patients. While this manuscript was in revision, Guo et $a l^{31}$ published online findings from a cohort of 12 primary prostatic small cell carcinomas and found that $67 \%$ contained $E R G$ rearrangements, with $88 \%$ (7/8) harboring ERG deletions. However, they did not address the presence of ERG rearrangement in concordant acinar tumors in this study, nor did they look at ERG protein expression in these tumors. Recently, Scheble et al also published the occurrence of ERG rearrangement in 86\% (12/14) cases of prostatic small cell carcinoma, a somewhat higher rate of rearrangement. ${ }^{32}$ In this study, we confirm and add to these data by characterizing the $E R G$ gene and protein status in the largest cohort of primary prostatic small cell carcinomas and their concurrent acinar tumors to date. In contrast to the high rate found by Han et al and Scheble et al and consistent with recent data obtained from the study by Guo et al, we found that the rate of $E R G$ rearrangement in primary prostatic small cell carcinoma is $45 \%$, a rate very similar to that documented in acinar carcinoma, which typically hovers between 40 and $60 \%$ in surgical cohorts. Similar to the studies by both Han et al and Guo et al, we did find that rearrangement by deletion is more common in small cell carcinomas, as rearrangement by ERG translocation occurred in a minority $(20 \%)$ of our rearranged cases. Overall, as ERG rearrangements are not enriched in this cohort of primary small cell carcinomas, our data are consistent with a number of recent studies indicating that $E R G$ rearrangement by itself is not a predictor of aggressive disease in prostate cancer. ${ }^{12,13}$ Although several studies have indicated that polysomy at the $E R G$ locus (with or without rearrangement) is a poor prognostic indicator, we were not able to reliably identify polysomy in this study because of the large number of overlapping nuclei in most small cell carcinoma cases. ${ }^{13,33}$

Despite the fact that small cell and acinar carcinomas often occur simultaneously, few previous studies have addressed the question of whether these two tumors are derived from a common clone. Hansel et $a l^{3}$ looked at a single case of concurrent acinar and small cell carcinomas and found a common TP53 mutation in the two components, with the small cell carcinoma showing a loss of the second wild-type allele, suggestive of clonal evolution. In the current study, we found that the majority of cases with concurrent small cell and acinar components showed concordance for ERG rearrangements, which lends support to the idea that the two tumor types share a common origin. However, these data do not resolve the question of whether the two tumors emerged separately from a common stem cell, or whether the small cell carcinoma evolved from the acinar adenocarcinoma. Further molecular studies will be necessary to resolve this issue. 
Recently, several groups have provided evidence that there is an etiological link between the androgen-signaling axis and $E R G$ rearrangement in prostate cancer. ${ }^{17-19}$ It has been known for some time that the $5^{\prime}$ partners for $E R G$ rearrangement are typically androgen-regulated genes (eg, TMPRSS2 and $S L C 45 A 3)$, and that the chromosomal rearrangement places the expression of ERG under androgen regulation. However, several recent papers have suggested that androgen signaling may actually have a causal role in these genomic rearrangements by inducing proximity of the involved loci and potentially promoting double-stranded breaks. ${ }^{17-19}$ Interestingly, small cell carcinomas are often unresponsive to androgen-deprivation therapies, and the small cell carcinoma cell line that harbors ERG rearrangement, NCI-H660 is AR negative. ${ }^{2,24-30}$ In the current study, we looked at two additional markers of the androgen-signaling axis, AR and an androgen-regulated prostate specific transcription factor, NKX3-1, which is known to function as a tumor suppressor in the prostate and is downregulated in some high Gleason grade tumors. ${ }^{20}$ Interestingly, AR and NKX3-1 were not expressed in the majority of small cell tumors examined, although the majority of the concurrent acinar foci were positive for these markers. Consistent with the fact that NKX3-1 is androgen regulated, small cell tumors that expressed ARs were typically also positive for NKX3-1.

The lack of AR expression in the majority of prostatic small cell carcinomas may also explain the low rate of ERG protein expression we observed in the ERG rearrangement-positive small cell carcinoma cases. As $E R G$ gene rearrangement frequently brings ERG under androgen-regulated transcriptional control, ERG protein expression in ERG rearrangement-positive tumors may provide an indirect readout of the androgen-signaling axis. Consistent with intact androgen signaling, in surgical cohorts of conventional acinar prostate carcinomas (those not associated with small cell carcinomas), ERG protein expression is typically $>90 \%$ sensitive for detection of $E R G$ gene rearrangement (AMD and GJN, unpublished data). We found a similarly high sensitivity in the small number of concurrent acinar carcinomas we examined in this study. In striking contrast, we found that the ERG protein was only expressed in $40 \%$ of ERG-rearranged small cell carcinomas. In addition, the lack of NKX3-1 expression in prostatic small cell carcinomas is also consistent with the notion that these tumors may no longer be reliant on androgen signaling. Interestingly, ERG rearrangements were more likely to be found in small cell tumors that did express AR and NKX3-1 than in those that did not express these markers; however, this trend was not statistically significant given the small number of AR-positive tumors. One might also speculate that the presence of ERG rearrangement in AR- and NKX3-1-negative tumors is consistent with the hypothesis that these tumors evolved from acinar tumors with active androgen signaling.

Finally, the current study validates ERG rearrangement as a potentially useful clinical marker of prostatic origin. Given that the small cell morphological phenotype can be encountered in tumors originating from various organs and that such tumors are often widely metastatic at presentation, identifying their primary site of origin presents a clinical challenge. Previous studies have established that prostate-specific immunostains (such as PSA, p501s and PSMA) are positive in only a minority of small cell cases (approximately 20-25\% ). ${ }^{4-6}$ Furthermore, TTF-1 is often positive in prostate small cell carcinomas, making it difficult to distinguish from a pulmonary small cell carcinoma. ${ }^{5,6}$ To date, ERG rearrangements have not been identified in any nonprostatic carcinomas, making this molecular marker specific for prostatic origin. ${ }^{15,31}$ In this study, we confirm these data as we did not find any evidence of $E R G$ gene rearrangement in bladder and lung small cell carcinoma cases. As the rate of $E R G$ rearrangement in small cell carcinomas is $<50 \%$ in our series, negativity for this marker would not rule out the prostatic origin for a metastatic small cell tumor. However, positivity for rearrangement could be taken as evidence of prostatic origin. Finally, given that ERG protein expression is not sensitive or entirely specific for detection of $E R G$ gene rearrangement in small cell carcinomas, our data suggest that ERG immunohistochemistry is not a good substitute for ERG FISH in this tumor subtype.

In summary, we report that $E R G$ gene rearrangements occur in prostatic small cell carcinomas at a rate comparable with that in acinar carcinomas and are typically concordant in concurrent acinar and small cell tumors. However, in contrast to their acinar counterparts, AR and NKX3-1 are positive in a minority of prostatic small cell carcinomas and ERG protein expression is only seen in a subset of prostatic small cell carcinoma cases with ERG gene rearrangement. Overall, these data support a common origin for these two tumors and identify a promising molecular marker for establishing prostatic origin in small cell carcinomas of unknown primary.

\section{Acknowledgement}

Funding for this research was provided by the Patrick C Walsh Prostate Cancer Research Fund.

\section{Disclosure/conflict of interest}

The authors declare no conflict of interest.

\section{References}

1 Epstein JI, Netto GN. Biopsy Interpretation of the Prostate. 2nd edn. Lippincott, Williams \& Wilkins: Philadelphia, PA, 2008. 
2 Palmgren JS, Karavadia SS, Wakefield MR. Unusual and underappreciated: small cell carcinoma of the prostate. Semin Oncol 2007;34:22-29.

3 Hansel DE, Nakayama M, Luo J, et al. Shared TP53 gene mutation in morphologically and phenotypically distinct concurrent primary small cell neuroendocrine carcinoma and adenocarcinoma of the prostate. Prostate 2009;69:603-609.

4 Helpap B, Kollermann J. Undifferentiated carcinoma of the prostate with small cell features: immunohistochemical subtyping and reflections on histogenesis. Virchows Arch 1999;434:385-391.

5 Yao JL, Madeb R, Bourne P, et al. Small cell carcinoma of the prostate: an immunohistochemical study. Am J Surg Pathol 2006;30:705-712.

6 Wang W, Epstein JI. Small cell carcinoma of the prostate. A morphologic and immunohistochemical study of 95 cases. Am J Surg Pathol 2008;32:65-71.

7 Tomlins SA, Rhodes DR, Perner S, et al. Recurrent fusion of TMPRSS2 and ETS transcription factor genes in prostate cancer. Science 2005;310:644-648.

8 Tomlins SA, Mehra R, Rhodes DR, et al. TMPRSS2:ETV4 gene fusions define a third molecular subtype of prostate cancer. Cancer Res 2006;66: 3396-3400.

9 Mehra R, Tomlins SA, Shen R, et al. Comprehensive assessment of TMPRSS2 and ETS family gene aberrations in clinically localized prostate cancer. Mod Pathol 2007;20:538-544.

10 Perner S, Demichelis F, Beroukhim $\mathrm{R}$, et al. TMPRSS2:ERG fusion-associated deletions provide insight into the heterogeneity of prostate cancer. Cancer Res 2006;66:8337-8341.

11 Demichelis F, Fall K, Perner S, et al. TMPRSS2:ERG gene fusion associated with lethal prostate cancer in a watchful waiting cohort. Oncogene 2007;26: 4596-4599.

12 Fine SW, Gopalan A, Leversha MA, et al. TMPRSS2ERG gene fusion is associated with low gleason scores and not with high-grade morphological features. Mod Pathol 2010;23:1325-1333.

13 Gopalan A, Leversha MA, Satagopan JM, et al. TMPRSS2-ERG gene fusion is not associated with outcome in patients treated by prostatectomy. Cancer Res 2009;69:1400-1406.

14 Hessels D, Smit FP, Verhaegh GW, et al. Detection of TMPRSS2-ERG fusion transcripts and prostate cancer antigen 3 in urinary sediments may improve diagnosis of prostate cancer. Clin Cancer Res 2007;13:5103-5108.

15 Scheble VJ, Braun M, Beroukhim R, et al. ERG rearrangement is specific to prostate cancer and does not occur in any other common tumor. Mod Pathol 2010;23:1061-1067.

16 Han B, Mehra R, Suleman K, et al. Characterization of ETS gene aberrations in select histologic variants of prostate carcinoma. Mod Pathol 2009;22:1176-1185.

17 Lin C, Yang L, Tanasa B, et al. Nuclear receptorinduced chromosomal proximity and DNA breaks underlie specific translocations in cancer. Cell 2009; 139:1069-1083.
18 Mani RS, Tomlins SA, Callahan K, et al. Induced chromosomal proximity and gene fusions in prostate cancer. Science 2009;326:1230.

19 Haffner MC, Aryee MJ, Toubaji A, et al. Androgeninduced TOP2B-mediated double-strand breaks and prostate cancer gene rearrangements. Nat Genet 2010;42:668-675.

20 Bethel CR, Faith D, Li X, et al. Decreased NKX3-1 protein expression in focal prostatic atrophy, prostatic intraepithelial neoplasia, and adenocarcinoma: association with gleason score and chromosome 8p deletion. Cancer Res 2006;66:10683-10690.

21 Stein ME, Bernstein Z, Abacioglu U, et al. Small cell (neuroendocrine) carcinoma of the prostate: etiology, diagnosis, prognosis, and therapeutic implications-a retrospective study of 30 patients from the rare cancer network. Am J Med Sci 2008;336:478-488.

22 Di Sant'Agnese PA, Cockett AT. The prostatic endocrine-paracrine (neuroendocrine) regulatory system and neuroendocrine differentiation in prostatic carcinoma: a review and future directions in basic research. J Urol 1994;152:1927-1931.

23 Wenk RE, Bhagavan BS, Levy R, et al. Ectopic ACTH, prostatic oat cell carcinoma, and marked hypernatremia. Cancer 1977;40:773-778.

24 Hindson DA, Knight LL, Ocker JM. Small-cell carcinoma of prostate. transient complete remission with chemotherapy. Urology 1985;26:182-184.

25 Tetu B, Ro JY, Ayala AG, et al. Small cell carcinoma of the prostate. Part I. A clinicopathologic study of 20 cases. Cancer 1987;59:1803-1809.

26 Moore SR, Reinberg Y, Zhang G. Small cell carcinoma of prostate: effectiveness of hormonal versus chemotherapy. Urology 1992;39:411-416.

27 Rubenstein JH, Katin MJ, Mangano MM, et al. Small cell anaplastic carcinoma of the prostate: seven new cases, review of the literature, and discussion of a therapeutic strategy. Am J Clin Oncol 1997;20:376-380.

28 Papandreou CN, Daliani DD, Thall PF, et al. Results of a phase II study with doxorubicin, etoposide, and cisplatin in patients with fully characterized small-cell carcinoma of the prostate. J Clin Oncol 2002;20:3072-3080.

29 Spiess PE, Pettaway CA, Vakar-Lopez F, et al. Treatment outcomes of small cell carcinoma of the prostate: a single-center study. Cancer 2007;110:1729-1737.

30 Mertz KD, Setlur SR, Dhanasekaran SM, et al. Molecular characterization of TMPRSS2-ERG gene fusion in the NCI-H660 prostate cancer cell line: a new perspective for an old model. Neoplasia 2007; 9:200-206

31 Guo CC, Dancer JY, Wang Y, et al. TMPRSS2-ERG gene fusion in small cell carcinoma of the prostate. Hum Path 2010;42:11-17.

32 Scheble VJ, Braun M, Wilbertz T, et al. ERG rearrangement in small cell prostatic and lung cancer. Histopathology 2010;56:937-943.

33 Attard G, Clark J, Ambroisine L, et al., Transatlantic Prostate Group. Duplication of the fusion of TMPRSS2 to ERG sequences identifies fatal human prostate cancer. Oncogene 2008;27:253-263. 